\title{
Pulsars as excellent probes for the magnetic structure in our Milky Way
}

\author{
JinLin Han \\ National Astronomical Observatories, Chinese Academy of Sciences, \\ DaTun Road 20A, ChaoYang District, Beijing 100012, China \\ email: hjl@nao.cas.cn
}

\begin{abstract}
In this invited talk, I first discuss the advantages and disadvantages of many probes for the magnetic fields of the Milky Way. I conclude that pulsars are the best probes for the magnetic structure in our Galaxy, because magnetic field strength and directions can be derived from their dispersion measures (DMs) and rotation measures (RMs). Using the pulsars as probes, magnetic field structures in the Galactic disk, especially the field reversals between the arms and interarm regions, can be well revealed from the distribution of RM data. The field strengths on large scales and small scales can be derived from RM and DM data. RMs of extragalactic radio sources can be used as the indication of magnetic field directions in the spiral tangential regions, and can be used as probes for the magnetic fields in the regions farther away than pulsars when their median RMs are compared with pulsar RMs.
\end{abstract}

Keywords. pulsars: general, ISM: magnetic fields, Galaxy: structure

\section{Introduction}

Magnetic fields permeate the interstellar medium on all scales, from the stellar scale of AU size to the galactic scale of tens of kpc. In general, magnetic fields are tangled by many physical process in the interstellar medium, for example, the localized processes of star formation and supernova explosion, the galactic processes of differential rotation, density wave and stream motion, so that the ever-existed large-scale magnetic fields are very distorted. When we try to understand the properties of magnetic fields in our Milky Way, we have to know the magnetic fields of different scales in different regions.

There are many probes for the magnetic fields in our Milky Way. The most widely used are the starlight polarization, the polarized emission of dust and clouds at millimeter and submillimeter wavelength, the Zeeman effect of spectral lines or maser line from clouds or clumps, the diffuse radio synchrotron emission from relativistic electrons in the interstellar magnetic fields, the Faraday rotation of extragalactic radio sources and pulsars. The first three are related to magnetic field in clouds, and the later two are related to the fields in the diffuse medium. Each probe measures only one of the three dimensional field components or their average or integration. We have to understand the advantages and disadvantages of these probes, and then "connect" all measurements to get the real structure of the Galactic magnetic fields.

\section{Advantage and disadvantage of different probes for magnetic fields}

Some of of the above mentioned probes are more suitable to reveal the localized magnetic fields, and some are more sensitive to the large-scale fields. Because of our location at the disk edge in the Milky Way, the best probes for the Galactic magnetic structure have to be able to detect the magnetic component parallel to the line of sight. 


\subsection{Starlight polarization}

Starlight is not polarized when it is radiated. It becomes polarized when it passes through the interstellar medium. It is slightly absorbed or scattered by interstellar dust grains which are preferentially aligned by interstellar magnetic fields. The farther the star is, the more extinction its light suffers on the path to us, and the higher the polarization of starlight. Starlight polarization is the measurement of the summed extinction due to dusts in the path, therefore it indicates the averaged magnetic field orientations in the sky plane, not directions.

The polarization of stars around an individual cloud can be used to trace the magnetic field orientation inside the cloud. When more and more stars are observed in the very deep Galactic disk, the data can not separate magnetic field orientations in every clouds at different distances which on average are generally aligned with the Galactic plane. Therefore, the starlight probes can not be used for the large-scale magnetic structure in the Galactic disk. They are good probes for the local halo fields if a large number of high-latitude distant stars are observed in wide sky area.

\subsection{Polarized emission of dust and clouds}

The polarized emission from the clouds or dust is quite localized feature, and can show the magnetic field orientations in the clouds perpendicular to the line of sight. There is evidence that the magnetic fields in clouds are related to the fields of larger scales ( $\mathrm{Li} \&$ Henning 2011). Similar to the starlight probes, even if the magnetic orientations of many clouds in the disk of the Milky way are observed, we cannot get the large-scale magnetic structure, because the field orientation of all clouds are aligned with the Galactic plane in general. In fact, the polarized emission of dust and clouds has already been seen clearly from the polarization maps of WMAP or Planck, which indicate such parallel fields to the Galactic plane.

\subsection{The Zeeman effect of spectral lines from clouds or clumps}

The line emission or absorption from the clouds or clumps with internal magnetic fields show Zeeman splitting of the line. The separation of the split lines measures the field strength of the magnetic component parallel to the line of sight, and the sense change of circular polarization of the split lines indicates the direction of the field component on the line of sight. Physically the measurements are sensitive only to the fields inside the clouds or clumps, which are of a scale of pc or even AU. Surprisingly, the distribution of available magnetic field measurements from maser lines around HII regions are very coherent with the large-scale spiral structure and large-scale magnetic fields (Han \& Zhang 2007). A big project using the ATCA (Green et al. 2012) is in progress for extensive observations of the Zeeman splitting of lines from HII regions for large-scale magnetic structure.

\subsection{Diffuse radio synchrotron emission and polarization}

The polarization of synchrotron radiation from relativistic electrons shows the average orientation of magnetic fields in the sky plane in the emission region, perpendicular to the line of sight. If random magnetic fields dominate in the emission region, as is often the case, then polarization "vectors" (orientation, not direction) could be very random, and the observed emission is depolarized. The polarized emission from different regions inside the Milky Way at different distances also suffers different Faraday rotations when it propagates in the interstellar medium. As we are located on the edge of the disk of the Milky Way, the observed synchrotron radiation is the superposition of such variously Faraday-rotated polarized radiation from everywhere at all distances until the Milky way 
"boundary", which could be therefore very depolarized. The lower the observational frequency, the stronger the depolarization. The closer towards the Galactic Center along the Galactic plane, the less ordered the polarization. In the outer region near the anticenter of the Milky way, the radio emission comes from only the Perseus arm, and we see more polarized emission than in the inner Galaxy (Xiao et al. 2011).

Using the polarization survey of radio synchrotron of the whole sky, one can get some constraints on the magnetic fields in the halo of Milky Way, but it is hard to constrain the magnetic fields in the Galactic disk. Because there is no linearity for Faraday rotation against distance, and because the similarly polarized emission can come from regions of different distances, the rotation measure synthesis cannot separate the polarized emission from different regions.

\subsection{Rotation measures of extragalactic radio sources}

Faraday rotation is the summed rotation of the polarization angle $\phi$ of a linearly polarized wave on the way from a source $\left(\phi_{0}\right)$ to us, $\phi=R M * \lambda^{2}+\phi_{0}$. The rotation measure (RM) is related to electron density and magnetic fields by $\mathrm{RM}=0.810 \int_{\text {source }}^{\text {observer }} n_{e} \mathbf{B} \cdot d \mathbf{l}$ (in unit of $\mathrm{rad} \mathrm{m}^{-2}$ ). Here $n_{e}$ is the electron density in $\mathrm{cm}^{-3}, \mathbf{B}$ is the vector magnetic field in $\mu \mathrm{G}$ and $d \mathbf{l}$ is an elemental vector along the line of sight toward us in pc. Obviously, the $\mathrm{RM}$ is sensitive to the magnetic field component on the line of sight, weighted by the electron density. Notice that it is an integrated value. Positive RMs correspond to the average fields on the path directed toward us. Random or smaller-scale fields on the path cannot be recognized from the final observed wavelength dependence of $\phi$.

There are many background extragalactic radio sources (EGRs) distributed in the sky, which can be the most powerful probe of the magnetic field in the halo of our Milky Way. The observed RMs contain three contributions: 1) the intrinsic RM from the source, which depends on the observational wavelength and how deep in the source the radiation comes from, 2) the RM of intergalactic medium which is probably very small and not measured yet, and 3) the RM foreground from our Milky Way. The RMs of a number of EGRs in a given sky region should have more or less the common foreground Galactic RM contribution. The large-scale RM sky distribution therefore shows the magnetic fields in the halo (Han et al. 1997, 1999). The RM sky of more dense data set (Taylor et al. 2009, Oppermann et al. 2012) can show the details of magnetic fields in visually large objects (Harvey-Smith et al. 2011), in addition to the general RM sky of large angular scales.

At lower Galactic latitudes, extensive efforts have recently been made to enlarge the RM samples (e.g. Brown et al. 2007, Van Eck et al. 2011). Towards the central region the data become more scarce, because the diffuse emission is stronger and because the polarization observations are more difficult to carry out. The median RMs of background EGRs behind the disk are the integrated measurement of polarization angle rotations over the whole path in the disk and therefore not sensitive to the possible magnetic field reversals between arms and interarm regions on the path. The dominant contribution to RMs of EGRs comes from tangential regions, where the magnetic fields have the smallest angle with the line of sight if the fields follow spiral arms. When a set of RMs of EGRs are fitted with a magnetic structure model, the electron density model is a necessary independent input.

\section{Pulsars as probes for the Galactic magnetic fields}

Pulsars are polarized radio sources inside our Milky Way. The observed RMs of pulsars come only from the interstellar medium between pulsars and us, because there is no intrinsic Faraday rotation from the emission region and pulsar magnetosphere (Wang 


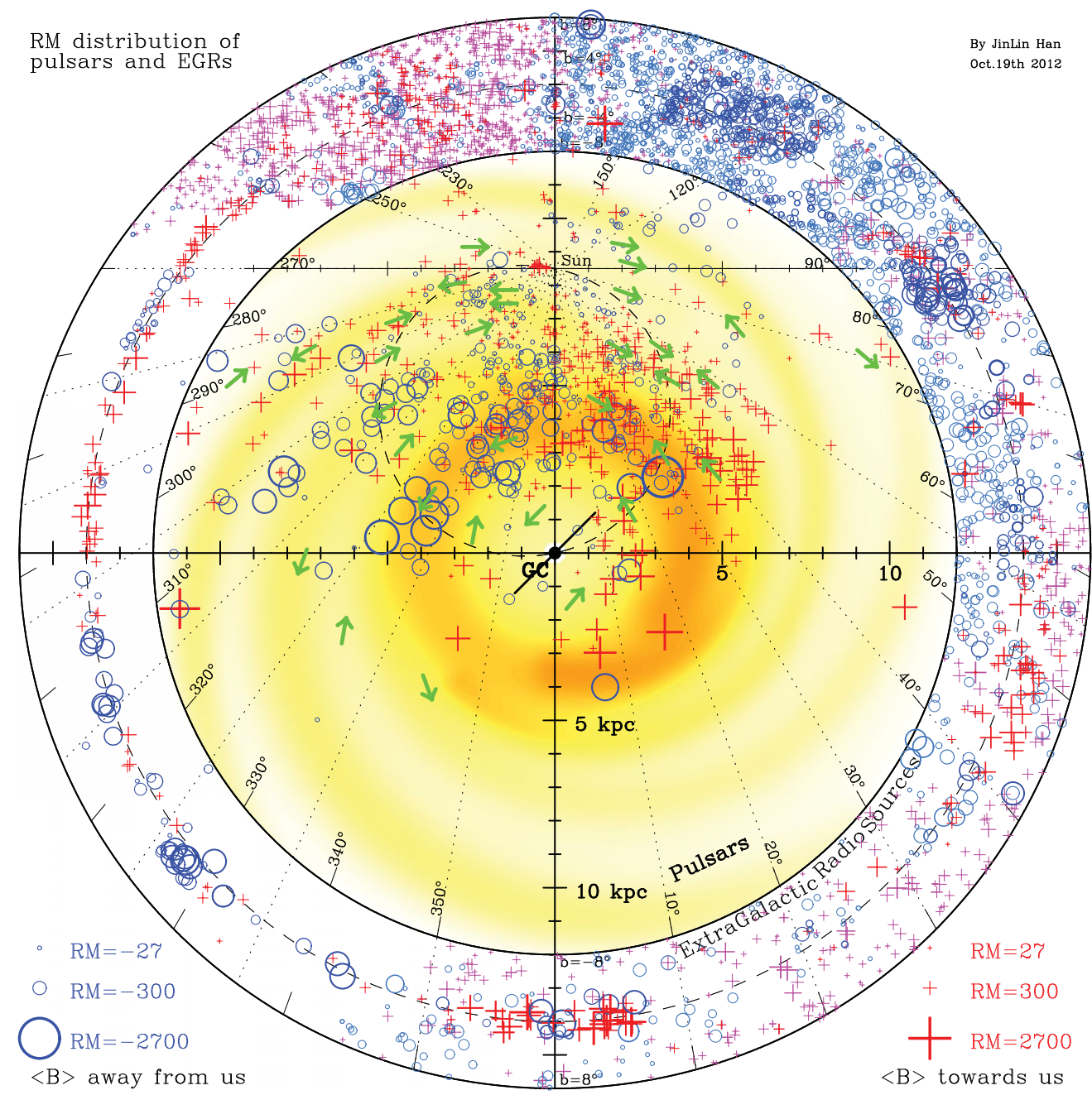

Figure 1. The RM distribution of 736 pulsars of $|b|<8^{\circ}$ projected onto the Galactic plane, including new data of Han et al (2012, in preparation). The linear sizes of the symbols are proportional to the square root of the RM values with limits of \pm 27 and $\pm 2700 \mathrm{rad} \mathrm{m}^{-2}$. Positive RMs are shown by plus signs and negative RMs by open circles. The background shows the approximate locations of spiral arms used in the NE2001 electron density model (Cordes \& Lazio 2002). Published RMs of EGRs of $|b|<8^{\circ}$ are displayed in the outer ring according to their $l$ and $b$, with the same convention of RM symbols and limits. The data from the NVSS RM catalog (Taylor et al. 2009) are plotted in light-blue and pink symbols. The large-scale structure of magnetic fields in the Galactic disk, as indicated by arrows, are derived from the distribution of pulsar RMs and the comparison of pulsar RMs with the RMs of background EGRs.

et al. 2011). For a pulsar at distance $D$ (in pc), the RM is given by $\mathrm{RM}=0.810 \int_{0}^{D} n_{e} \mathbf{B} \cdot d \mathbf{l}$. With the pulsar dispersion measure, $\mathrm{DM}=\int_{0}^{D} n_{e} d l$, we obtain a direct estimate of the field strength weighted by the local free electron density

$$
\left\langle B_{\|}\right\rangle=\frac{\int_{0}^{D} n_{e} \mathbf{B} \cdot d \mathbf{l}}{\int_{0}^{D} n_{e} d l}=1.232 \frac{\mathrm{RM}}{\mathrm{DM}} .
$$

Pulsars are spread through the Galaxy at approximately known distances, allowing threedimensional mapping of the magnetic fields. If pulsar RM data are model-fitted with the 
magnetic field structures with the electron density model (Han \& Qiao 1994), then the pulsars and EGRs are more or less equivalently good as probes for the magnetic structure. But when RM and DM data are available for many pulsars in a given region with similar lines of sight, e.g. one pulsar at $d 0$ and one at $d 1$, the RM change against distance or DM can indicate the direction and magnitude of the large-scale field in particular regions of the Galaxy (Han et al. 1999, 2002, 2006). Field strengths in the region can be directly derived by using

$$
\left\langle B_{\|}\right\rangle_{d 1-d 0}=1.232 \frac{\Delta \mathrm{RM}}{\Delta \mathrm{DM}},
$$

where $\left\langle B_{||}\right\rangle_{d 1-d 0}$ is the mean line-of-sight field component in $\mu \mathrm{G}$ for the region between distances $d 0$ and $d 1, \Delta \mathrm{RM}=\mathrm{RM}_{d 1}-\mathrm{RM}_{d 0}$ and $\Delta \mathrm{DM}=\mathrm{DM}_{d 1}-\mathrm{DM}_{d 0}$. Notice that this derived field is not dependent on the electron density model.

As shown in Han et al. (2006), the available pulsar RM data show that magnetic fields in the spiral arms (i.e. the Norma arm, the Scutum and Crux arm, and the Sagittarius and Carina arm) are always counterclockwise in both the first and fourth quadrants, though some disordered fields appear in some segments of some arms. At least in the local region and in the fourth quadrant, there is good evidence that the fields in interarm regions are similarly coherent, but reversed to be clockwise. Therefore at least four or five reversals in the fourth quadrant occur from the centre to the outskirts of our Milky Way. In the central Galactic region interior to the Norma arm, new RM data of pulsars indicate that the fields are clockwise, reversed again from the counterclockwise field in the Norma arm. In the first Galactic quadrant, because the separations between spiral arms are so small, the RM data are dominated by counterclockwise fields in the arm regions though a few negative pulsar RMs indicate clockwise fields in the interarm regions.

We notice that the averaged variation of RMs of extragalactic radio sources along the Galactic longitudes (Brown et al. 2007) are consistent with the field reversal pattern obtained from pulsar RMs.

Using pulsar RM and DM data, Han et al. (2006) were able to measure the strength of regular azimuthal fields near the tangential regions in the 1st and 4th Galactic quadrants. Although the "uncertainties", which in fact reflect the random fields, are large, the tendency is clear that fields get stronger at smaller Galactocentric radius and weaker in interarm regions. The radial variation is,

$$
B_{\text {reg }}(R)=B_{0} \exp \left[\frac{-\left(R-R_{\odot}\right)}{R_{\mathrm{B}}}\right],
$$

with the strength of the large-scale field at the Sun, $B_{0}=2.1 \pm 0.3 \mu \mathrm{G}$, and the scale radius $R_{\mathrm{B}}=8.5 \pm 4.7 \mathrm{kpc}, R$ is the distance from the Galactic center, $R_{\odot}=8.5 \mathrm{kpc}$ is the galactocentric distance of the Sun.

Pulsar RMs have also been used to study the small-scale random magnetic fields in the Galaxy. Some pairs of pulsars close in sky position have similar DMs but very different RMs, indicating an irregular field structure on scales of about 100 pc. Some of these irregularities may result from HII regions in the line of sight to a pulsar (Mitra et al. 2003). It has been found from pulsar RMs that the random field has a strength of $B_{r} \sim 4-6 \mu \mathrm{G}$ independent of cell-size in the scale range of $10-100$ pc. From pulsar RMs in a very large region of the Galactic disk, Han et al. (2004) obtained a power law distribution for magnetic field fluctuations of $E_{B}(k)=C\left(k / \mathrm{kpc}^{-1}\right)^{-0.37 \pm 0.10}$ at scales from $1 / k=$ $0.5 \mathrm{kpc}$ to $15 \mathrm{kpc}$, with $C=(6.8 \pm 0.8) 10^{-13} \mathrm{erg}^{-3} \mathrm{kpc}$, corresponding to an $\mathrm{rms}$ field of $\sim 6 \mu \mathrm{G}$ in the scale range. 


\section{Conclusions and discussions}

Pulsars are excellent probes of the magnetic fields in our Milky Way. They are the best to reveal the magnetic field structure in the Galactic disk, especially the field reversals; they are the best to derive the magnetic field strength - much less model-dependent than other probes; and they are the best to get the observational spatial energy spectrum of the magnetic fields. Pulsars can also be used to probe the magnetic fields in the Galactic halo. We already have RMs for about half of the known pulsars and these have been used for studies of the Galactic magnetic fields. In the future, when more and more known pulsars are observed for their rotation measures, we can get more details of magnetic structure in the nearby half of the Galactic disk.

Note that magnetic fields in the Galactic disk of the far side of the Galactic center are not yet explored; very few distant pulsars have been found there. When more and more pulsars are discovered in the far half of the disk, using FAST and SKA, for example, we can study the differences in RMs and DMs of pulsars at various distances in different arms, and measure the magnetic field directions and strength in the remote arms, so that the global structure of the disk fields can be well revealed. However, if the largescale magnetic fields always go along arms, as present data suggest, the RMs of distant pulsars will become less sensitive to the magnetic fields in the far half disk because the lines of sight will be more perpendicular to the spiral arms than in the nearby half. The distribution of the magnetic fields of $\mathrm{OH}$ masers in HII and star formation regions can always be used as supplementary tools for large-scale magnetic fields (Han \& Zhang 2007), if the large-scale fields of the large-scales are somehow "remembered" in clouds or clumps of small scales as currently available data suggested.

\section{Acknowledgements}

Thanks for reading the manuscript go to Dr. Dick Manchester, who has cooperated with me for many years on the topic. The author is supported by the National Natural Science Foundation of China (10833003).

\section{References}

Brown, J. C., Haverkorn, M., Gaensler, B. M., et al. 2007, ApJ, 663, 258

Cordes, J. M. \& Lazio, T. J. W. 2002, preprint (arXiv:astro-ph/0207156)

Green, J. A., McClure-Griffiths, N. M., Caswell, et al. 2012, MNRAS, 525, 2530

Li, H. B. \& Henning, T. J. 2011, Nature, 479, 499

Han, J. L. \& Qiao, G. J. 1994, A\&A, 288, 759

Han, J. L., Beck, R., Ehle, M., Haynes, R. F., \& Wielebinski, R. 1999a, A\&JA, 348, 405

Han, J. L., Ferriere, K., \& Manchester, R. N. 2004, ApJ, 610, 820

Han, J. L., Manchester, R. N., Berkhuijsen, E. M., \& Beck, R. 1997, A\&SA, 322, 98

Han, J. L., Manchester, R. N., \& Qiao, G. J. 1999, MNRAS, 306, 371

Han, J. L., Manchester, R. N., Lyne, A. G., \& Qiao, G. J. 2002, ApJ, 570, L17

Han, J. L., Manchester, R. N., Lyne, A. G., Qiao, G. J., \& van Straten, W. 2006, ApJ, 642, 868.

Han, J. L. \& Zhang, J. S. 2007, A\& A, 464, 609

Harvey-Smith, L., Madsen, G. J., \& Gaensler, B. M. 2011, ApJ, 736, 83

Mitra, D., Wielebinski, R., Kramer, M., \& Jessner, A. 2003, A\& A, 398, 993

Oppermann, N., Junklewitz, H., Robbers, G. et al. 2012, A\&3A, 542, A93

Taylor, A. R., Stil, J. M., \& Sunstrum, C, 2009, ApJ, 702, 1230

Van Eck, C. L., Brown, J. C., Stil, J. M. et al. 2011, ApJ, 728, 97

Wang, Chen, Han, J. L. \& Lai, Dong 2011, MNRAS, 417, 1183

Xiao, L., Han, J. L., Reich, W., et al. 2011, A\&BA, 529, A15 